\title{
Safety of a new conjugate meningococcal C vaccine in infants
}

\author{
R Lakshman, I Jones, D Walker, K McMurtrie, L Shaw, G Race, S Choo, L Danzig, \\ P Oster, A Finn
}

\begin{abstract}
Background-Group C conjugate meningococcal vaccines (Men $C$ ) were introduced into the UK primary immunisation schedule in November 1999. There has been extensive professional and public interest in their efficacy and safety.

Aim-To determine the occurrence of at least one uncommon adverse event in infants related to the administration of the Chiron Men C vaccine.

Methods-A total of 2796 infants aged approximately 2 months were recruited into the study from areas in and around Sheffield and from Scotland. They were vaccinated with the Chiron Men $C$ vaccine at 2,3 , and 4 months along with routine immunisations. Data on adverse events occurring one month after each dose were collected actively and prospectively and reviewed for possible relation to the vaccine.

Results-There were no deaths. There were no serious adverse events considered definitely or probably caused by the vaccine. Four infants developed serious adverse events (hypotonia, screaming syndrome, maculopapular rash, and agitation, respectively) that were considered possibly related to the vaccine. All recovered completely. Adverse events were seen in 1804 children but were considered possibly related to the vaccine in only 49 $(1.8 \%)$. On subsequent immunisation there were no recurrences of adverse events considered to be possibly related to the vaccine.

(Arch Dis Child 2001;85:391-397)
\end{abstract}

Keywords: vaccine; meningococcal; Men C; meningitis; immunisation; adverse reaction

Meningococcal septicaemia and meningitis are important causes of morbidity and mortality in the UK and many other parts of the world. The predominant serogroups responsible for infection in the UK are B and C. In the year ending June 2000, there were 889 laboratory confirmed cases of group $\mathrm{C}$ meningococcal disease in England and Wales. ${ }^{1}$ The case fatality rate has remained at over $10 \%$ and is responsible for a high level of public anxiety. This has led to the interest in developing effective vaccines against the organism, even though the overall burden caused by meningococcal disease is low. Studies involving group C conjugate meningococcal vaccines have shown that when three doses of these vaccines are administered in infancy, they induce good immune responses and also prime for a booster response suggestive of immunologic memory. ${ }^{2-7}$ Conjugate meningococcal serogroup $C$ vaccines have been licensed in the UK and were adopted into the universal immunisation in November 1999, leading to dramatic reductions in disease caused by meningococcus C. ${ }^{8}$

Before introducing any new vaccine into general use, safety data must be collected. The issue of the appropriate design and size of prelicensure vaccine trials is topical. The recent withdrawal in the USA of live oral reassortant rotavirus vaccine as a result of its association with intussuception ${ }^{9}$ emphasises both the need for large prelicensure safety studies and the importance and effectiveness of postlicensure safety monitoring. Adverse events following vaccination may be true adverse reactions or may be temporally associated with the vaccine only by coincidence. Loss of confidence in a vaccine can lead to decreased coverage, return of epidemic disease, and avoidable deaths. Research in vaccine safety can help distinguish true vaccine reactions from coincidental events. Prelicensure trials which involve small sample sizes (up to several hundred subjects) are useful to observe vaccine reactogenicity but will not identify uncommon adverse events.

Between October 1998 and March 2000 we conducted a large field trial into the safety of the Chiron Men C vaccine in infants. Studies involving small numbers of subjects had shown this vaccine to be immunogenic and well tolerated in infants, ${ }^{6}$ toddlers, ${ }^{10}$ and adolescents. ${ }^{11}$ This study aimed to identify at least one uncommon adverse event associated with administration of this vaccine in infancy.

\section{Methods}

ETHICAL APPROVAL

Ethical approval for the study was obtained from the Trent multicentre research ethics committee and local research ethics committees in Sheffield and surrounding areas (Barnsley, Rotherham, Doncaster, North Derbyshire, North Nottinghamshire and Bassetlaw) and in Scotland (Tayside, Lanarkshire, and Ayrshire \& Arran).

\section{STUDY POPULATION AND INCLUSION AND} EXCLUSION CRITERIA

Healthy male and female infants $(n=2796)$ aged approximately 2 months, who were to receive their routine infant immunisations, were recruited into this open label study from two study centres in the UK. Subjects were enrolled in general practitioners' surgeries in 
Table 1 Definition of categories of relation of adverse event to study vaccine administration

\begin{tabular}{ll}
\hline Relation & Definition \\
\hline Not related & $\begin{array}{l}\text { The adverse event is obviously explained by another cause and/or the time } \\
\text { of occurrence of the adverse event is not reasonably related to the } \\
\text { vaccination } \\
\text { The adverse event is likely to be explainable by causes other than } \\
\text { vaccination } \\
\text { Vaccine administration and the adverse event occurrence are reasonably } \\
\text { related in time and the adverse event is explained equally well by causes } \\
\text { other than vaccination } \\
\text { Vaccine administration and the adverse event are temporally related and the } \\
\text { event is more likely to be explained by the vaccine than by other } \\
\text { mechanisms }\end{array}$ \\
Probably related & $\begin{array}{l}\text { Vaccine administration and the adverse event are temporally related and the } \\
\text { adverse event is consistent with the pattern of other known vaccine related } \\
\text { events }\end{array}$ \\
\hline
\end{tabular}

Sheffield and neighbouring communities ( $\mathrm{n}=1484$, of 2521 eligible infants offered information) and in the Ayrshire, Lanarkshire, and Tayside areas of Scotland $(n=1312$, of 2388 eligible infants offered information). Seventy eight surgeries in and around Sheffield and 87 in Scotland took part. Children who withdrew from the study were not replaced.

Parents or legal guardians gave written informed consent for inclusion into the study and confirmed that they would be available for the duration of the study (three months). Infants had to be in good health, born after 36 weeks' gestation, and with a birth weight of at least $2.5 \mathrm{~kg}$. Those who had contraindications to immunisation and those who had received any prior immunisation were excluded. In addition, infants who had suffered from suspected or proven meningococcal C infection or who had experienced household exposure or intimate contact with an individual with meningococcal $\mathrm{C}$ infection in the previous 60 days were not included in the study.

INTERVENTIONS AND THEIR TIMING

Enrolled infants received Men $\mathrm{C}$ vaccine (Chiron meningococcal $\mathrm{C}$ conjugate vaccine containing $10 \mu \mathrm{g}$ of meningococcal $\mathrm{C}$ oligosaccharide conjugated with $12.5-33.3 \mu \mathrm{g}$ of diphtheria toxoid, CRM197) which was given intramuscularly into the anterolateral area of the right thigh at 2,3 , and 4 months (the protocol allowed an interval range of 28-42 days between vaccinations). Routine vaccinations administered were commercially available diphtheria, tetanus, and whole cell pertussisHaemophilus influenzae type $\mathrm{b}$ conjugate (DTwP-Hib, Aventis Pasteur or Evans/ SmithKline Beecham or Behring/Cynamid) given concomitantly by intramuscular injection into the left thigh and oral polio vaccine (SmithKline Beecham).

PRIMARY OUTCOME MEASURES

The main outcome studied was adverse events occurring within 30 days of administration of the vaccine. Adverse events necessitating a visit to a doctor, all serious adverse events, and adverse events resulting in early exit from the study were collected at each vaccination visit, starting with the initial vaccination and by telephone 28-42 days after the third vaccination.

Any untoward occurrence necessitating a visit to a doctor was defined as an adverse event irrespective of causality. This included visits made for common childhood complaints. The severity of adverse events was classified as mild (no change in daily behaviour), moderate (some change in daily behaviour), or severe (significant change in daily behaviour). A serious adverse event was defined as any experience that suggested a significant hazard, contraindication, side effect, or precaution. It includes death, life threatening reactions, hospitalisation or prolongation of hospitalisation, persistent or significant disability or incapacity, or any event that for other reasons was considered medically significant. Whether adverse events were likely to have been caused by vaccination was judged by the investigators (either the general practitioner who assessed the child, or, in the case of hospital admissions and other serious adverse events by the principal investigators) to fall into one of five categories (see table 1).

All infants with identified adverse events were followed up until resolution or until a cause was identified. For events which remained unresolved at the end of the study, clinical assessments were made as to how long continued follow up was necessary.

\section{SAMPLE SIZE CALCULATIONS}

Initially it was planned to recruit a total of 2500 infants to the study. This would have given a $92 \%$ chance of detecting at least one uncommon adverse event (defined as an adverse event occurring at the rate of 1 per 1000) and a $22 \%$ chance of observing at least one rare adverse event (defined as occurrence at the rate of 1 per 10 000). However actual enrolment was 2796 infants which gave a $94 \%$ and $24 \%$ chance of detecting uncommon and rare adverse events, respectively.

\section{STATISTICAL ANALYSIS}

Baseline characteristics such as gestational age, birth weight, and sex were tabulated and analysed. The primary safety variables were adverse events necessitating a physician visit, serious adverse events, and adverse events leading to early exit from the study. The number and percentage of subjects experiencing different adverse events were tabulated. If a subject experienced multiple adverse events related to the same condition, the adverse event was counted only once and the maximal severity and causality was recorded. Serious adverse events were listed and adverse events leading to early exit from the study were summarised separately. Safety data were included on all subjects, despite protocol deviations, until an individual was withdrawn from the study.

\section{Results}

\section{SUBJECT CHARACTERISTICS}

Table 2 summarises the demographic and baseline characteristics of the children. One hundred and ten subjects withdrew from the study before completion. Thus, safety data were available and analysed for 2796 subjects after the first immunisation, 2768 after the second immunisation, and $2725(96 \%)$ after the third immunisation (see fig 1). Two hundred and fifty 
Table 2 Summary of demographic characteristics of study subjects

Age at first immunisation Sex of infant

Ethnic origin

Gestational age at birth

Birth weight

Median 61 days (range $42-180$ )

1418 male $(51 \%), 1378$ female $(49 \%)$

White, non-hispanic 2707 (97\%), others $89(3 \%)$

Median 40 weeks (range $34-44$ )

Median $3.4 \mathrm{~kg}$ (range 1.6-5.5)

Ranges for birth weight and gestational age are outside that defined in the inclusion criteria as all infants (including those who deviated from protocol) are included in the safety analysis.

seven protocol deviations were identified. (In 145 subjects, protocol procedures were not followed (most commonly omission of preimmunisation temperature/administration of vaccine in wrong location); 49 subjects had incorrect treatment or dose (most commonly Men C not given concomitantly with other vaccines); in 33 subjects the inclusion or exclusion criteria were not met (most commonly lower birth weight/ shorter length of gestation); 15 were unable to complete the study as a result of study closure (necessitated by the introduction of the UK meningococcal $\mathrm{C}$ vaccine campaign); there were nine informed consent issues (timing of signing of the form); five had an excluded vaccine (for example, DTaP); and one was found to have a withdrawal criterion (Turner's syndrome) but was not withdrawn.)

ADVERSE EVENTS SUMMARY

Figure 2 summarises the adverse events. A total of 1804 infants $(65 \%)$ had adverse events. Forty nine $(1.8 \%)$ experienced 58 adverse events that were considered possibly, probably, or definitely related to the meningococcal $\mathrm{C}$ vaccine, of which four $(0.15 \%)$ were reported as serious adverse events. All children recovered. Six subjects withdrew prematurely because of adverse events that were considered possibly, probably, or definitely related to the vaccine. After subsequent immunisation there were no recurrences of adverse events considered to be possibly related to the vaccine.

\section{ADVERSE EVENTS LEADING TO PREMATURE} WITHDRAWAL

Nine infants were withdrawn prematurely because of adverse events; in six the adverse events were judged to be possibly, probably, or definitely related to the study vaccine (described below). Another three infants who had

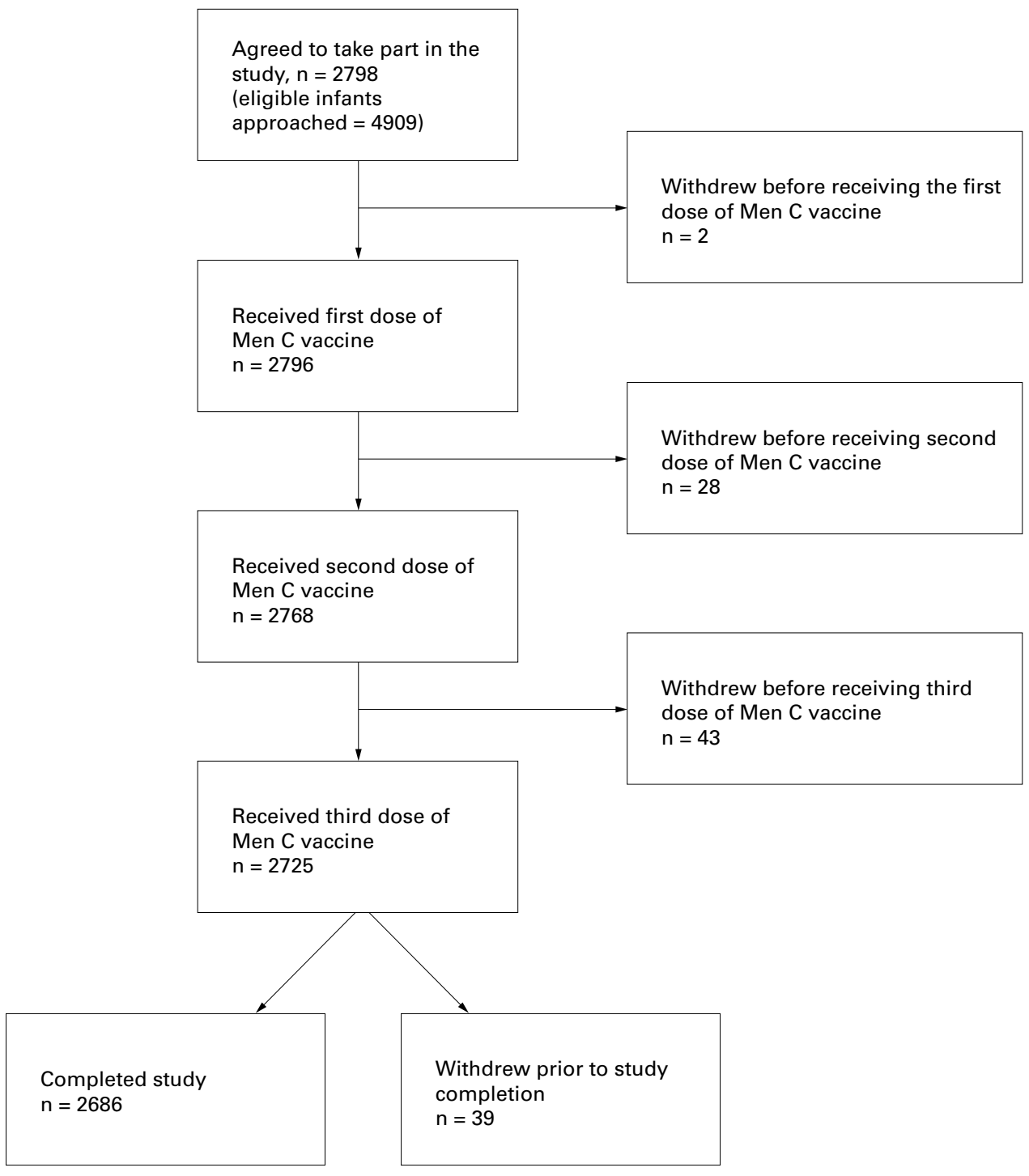

Figure 1 Flow diagram of study. 
consent withdrawn had an adverse event as a secondary reason for early exit from the study.

In one case, the adverse event was serious, namely a hypotonic hyporesponsive episode which was considered possibly related to the vaccine (details included in serious adverse events section below). Four infants were withdrawn after the first immunisation because of injection site reaction $(\mathrm{n}=1)$ or screaming syndrome $(n=3)$. Of these three, one case was associated with fever and diarrhoea and another with an injection site reaction. One subject was withdrawn after the second immunisation because of agitation.

SERIOUS ADVERSE EVENTS

There were no deaths. One hundred and thirty eight infants $(5 \%)$ had 161 serious adverse events (see table 3). All of these were associated with hospital admission. No serious adverse event was judged to be definitely or probably related to the vaccine. Four serious adverse events were considered possibly related to the study vaccine: hypotonia, screaming syndrome, maculopapular rash, and agitation.

A 57 day old boy developed a hypotonic hyporesponsive episode four hours after receiving his first dose of Men C and other routine immunisations. He suddenly became floppy and grey/blue around his lips. He regained tone after about five minutes but remained sleepy. $\mathrm{He}$ recovered completely but was withdrawn from the study.

A 64 day old girl developed moderate non-specific screaming a few hours after receiving her first dose of Men $\mathrm{C}$ and other routine immunisations. The screaming episode lasted longer than six hours but she recovered

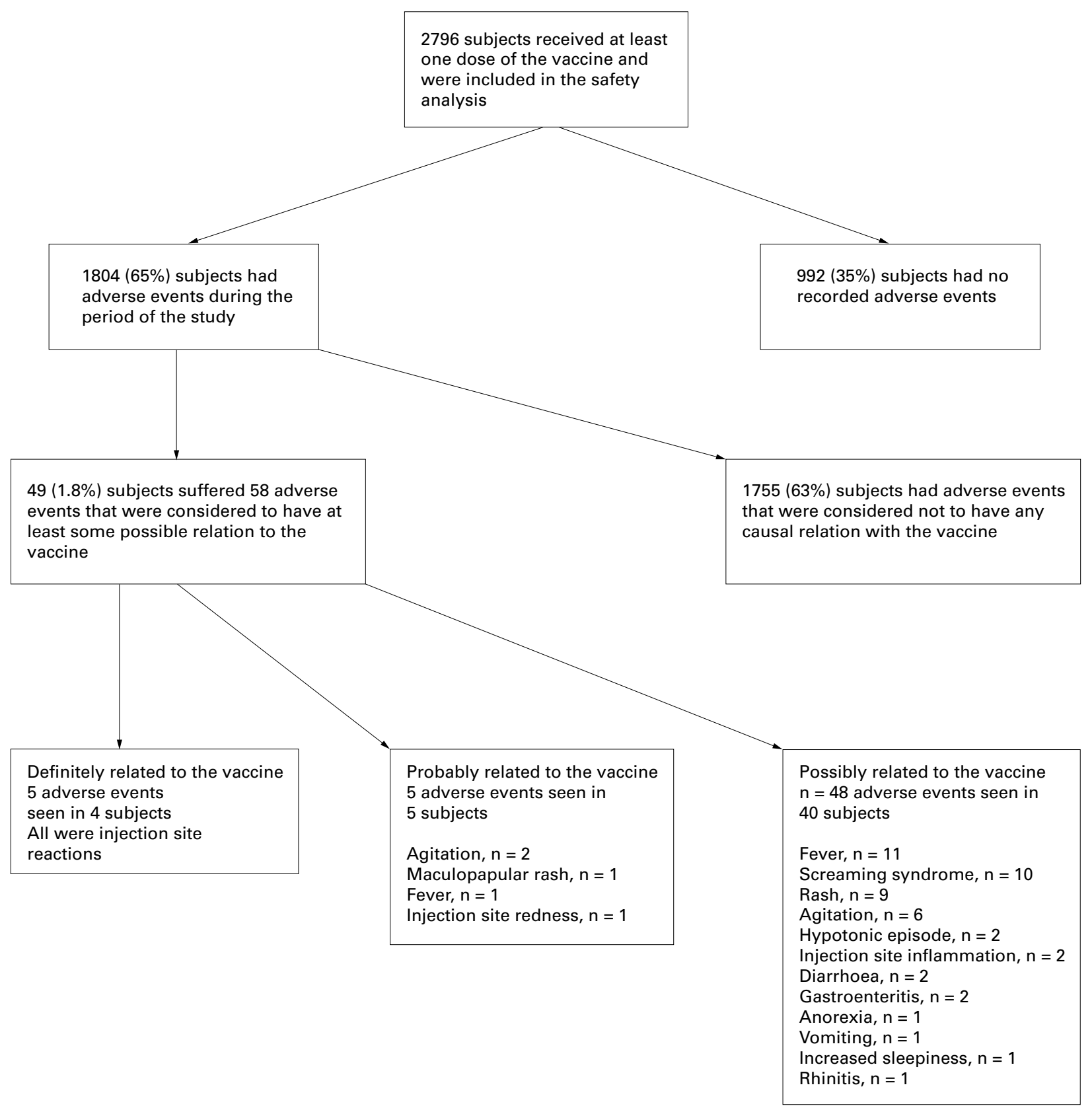

Figure 2 Summary of adverse events. 
Table 3 Serious adverse events

\begin{tabular}{|c|c|c|}
\hline Serious adverse event & \multicolumn{2}{|c|}{ Number of events } \\
\hline Viral respiratory tract infection & 45 & \\
\hline Gastritis and gastroenteritis & 23 & \\
\hline Bronchiolitis & 20 & \\
\hline Accidental injury or overdose & 9 & \\
\hline Elective surgical treatment/EUA & 10 & \\
\hline Gastro-oesophageal reflux & 6 & \\
\hline Anorexia and feeding problems & 4 & \\
\hline Constipation & 2 & \\
\hline Dysphagia & 1 & \\
\hline Vomiting & 1 & \\
\hline Periorbital cellulitis & 1 & \\
\hline Pertussis & 1 & \\
\hline Colic & 2 & \\
\hline Injection site haemorrhage & 1 & not at site of Men C injection \\
\hline Pneumonia & 1 & \\
\hline Intussusception & 2 & \\
\hline Tonsillitis & 1 & \\
\hline Agitation & 3 & 1 possibly related to Men $C$ vaccine \\
\hline Convulsions & 3 & \\
\hline Apnoea & 3 & \\
\hline Suspected sepsis & 2 & \\
\hline Maculopapular rash & 3 & 1 possibly related to Men $\mathrm{C}$ vaccine \\
\hline Purpuric rash & 3 & \\
\hline Urinary tract infection & 5 & \\
\hline Asthma and wheezing & 2 & \\
\hline Hypotonia & 1 & possibly related to Men $\mathrm{C}$ vaccine \\
\hline Chicken pox & 1 & \\
\hline Screaming syndrome & 1 & possibly related to Men $\mathrm{C}$ vaccine \\
\hline Peripheral oedema & 1 & \\
\hline Lipoma & 1 & \\
\hline Non-accidental injury & 1 & \\
\hline Unable to hear (parental report) & 1 & normal hearing on testing \\
\hline Total & 161 & \\
\hline
\end{tabular}

EUA, examination under anaesthesia.

completely (and continued in the study). This problem did not recur with subsequent immunisations.

A boy who was nearly 4 months old became unwell on the day he was given his third dose of Men C and routine immunisations. He developed fever and was irritable and fed less than normal. Four days later he developed a red maculopapular rash on his trunk. The provisional diagnosis was viral infection and he recovered completely in seven days. However, in view of the temporal relation to administration of the vaccine, an association could not be ruled out, so this adverse event was considered possibly related to the vaccine.

A girl who was just over 3 months old became unwell a day after receiving her second Men $\mathrm{C}$ and routine immunisations. She was irritable, crying intermittently and pulling at one ear. Examination was normal and she recovered without any specific treatment. She continued in the study and did not develop any adverse reaction to her third dose of Men C and routine immunisations.

ADVERSE EVENTS (DEFINITELY RELATED)

The four adverse events considered to be definitely related to the vaccine were all injection site reactions (redness, oedema, and/or swelling). One infant developed moderate injection site swelling and bruising after his first dose of the Men $\mathrm{C}$ vaccine. He was not given further doses of the vaccine. Another infant had a mild injection reaction to his second dose of vaccine but did not receive a further dose as he also developed moderate agitation after this dose. The other two infants developed mild local reactions (injection site oedema in the first and inflammation in the second) after their third dose. Thus none of these infants received further doses of the vaccine. The local reactions lasted for two days in two infants, and for 14 days in one. Information regarding duration could not be obtained in the fourth child.

ADVERSE EVENTS (PROBABLY RELATED)

Five infants experienced events that were considered probably related to the vaccine. Two developed agitation for about 24 hours after the vaccine. This occurred after the first dose in one infant and after the second dose in the other. The reaction was considered to be mild, so both infants went on to have subsequent doses of the vaccine. They did not become unwell with these doses. One child developed mild fever, which lasted less than a day after his second dose and continued in the study. Two children developed skin reactions at the site of injection: one had a maculopapular eruption which lasted for two days and occurred after the third dose; the other child developed a red spot which was about $5 \mathrm{~cm}$ in diameter and occurred after his second dose. The latter continued in the study and received his third dose of vaccine with no recurrence of this or any other adverse event.

\section{ADVERSE EVENTS (POSSIBLY RELATED)}

Forty eight adverse events were considered possibly related to the vaccine. These consisted of agitation $(n=6)$, screaming syndrome $(\mathrm{n}=10)$, rash $(\mathrm{n}=9)$, fever $(\mathrm{n}=11)$, diarrhoea $(n=2)$, gastroenteritis $(n=2)$, vomiting $(n=1)$, rhinitis $(n=1)$, anorexia $(n=1)$, injection site inflammation $(n=2)$, hypotonic episode $(n=2)$, and increased sleepiness $(\mathrm{n}=1)$.

Mild to moderate fever considered possibly related to the vaccine was seen in 11 infants. Men $C$ vaccination was stopped in one infant who also had screaming syndrome and diarrhoea with his fever. Two developed fever after their third dose of vaccine. The other eight received subsequent doses of vaccine without any ill effects. Agitation considered possibly related to the vaccine was seen in six. Four of these received further doses of the vaccine; none had recurrence of this or similar adverse event. The third dose of vaccine was not given to one infant who developed moderate agitation after his second dose. One infant did not receive further doses of vaccine as he developed agitation only at the time of his third dose. Screaming syndrome (defined as inconsolable crying lasting more than four hours and occurring within 48 hours after immunisation) occurred in 10 infants. It was of moderate severity in eight and severe in two. In seven it occurred after the first dose, in one after the second, and in two after their third immunisation. Infants who developed severe screaming syndrome were not given further doses of vaccine. Two infants developed screaming syndrome after their third dose. The remaining six continued in the study and received subsequent doses of vaccine with no ill effects. Nine infants developed a rash that was considered possibly related to the vaccine. None of the 
rashes that were considered to be possibly related to the vaccine were described as urticarial or purpuric.

\section{Discussion}

The likelihood of adverse events caused by vaccines can to some extent be predicted from experience with other related vaccines. Polysaccharide vaccines containing meningococcal $\mathrm{C}$ capsular polysaccharide have been safely used for many years. Haemophilus influenzae type b (Hib) vaccines in routine use are made by conjugation of the polyribosyl ribitol phosphate (the Hib capsular polysaccharide) to a diphtheria or tetanus toxoid protein. Thus it is biologically likely that Men $\mathrm{C}$ conjugate vaccines made by conjugation of Men C capsular polysaccharide conjugated either to diphtheria or tetanus toxoids will also be safe.

Safety studies such as this are designed systematically to record all adverse events that are temporally related to the study drug or vaccine in order to make them as sensitive as possible. Only a minority of adverse events that occur in temporal association with a vaccine are causally related. An optimal design for this study would thus have included a comparison control group of infants, allocated blindly and randomly to receive routine immunisations and a placebo injection. However, administration of placebo injections to infants is not generally considered ethically acceptable. Use of another active vaccine in a control group could confound results. Inclusion of a comparison group would have improved study design but has significant logistical difficulties and financial implications. There would also have been logistical difficulties in maintaining motivation and interest among families of infants selected as controls. For these reasons an open label single group design was used with careful comprehensive prospective collection of adverse event data. In order to attempt to differentiate coincidental events from related events, clinical characteristics of each event were studied looking for possible alternative causes. They were also considered in light of previous experience with this or similar vaccines in humans and animals.

In this study adverse events were common (seen in 1804 subjects, 65\%), but most were not assessed to be related to the vaccine (possibly, probably, or definitely related, 49 subjects, $2 \%)$. Serious adverse events possibly related to the vaccine were observed only in four $(0.15 \%)$ infants. Three of these (screaming syndrome, hypotonic episode, and agitation) are well recognised to be associated with DTP (diphtheria, tetanus, whole cell pertussis) immunisation, which was given along with the Men $C$ vaccine in this study. Decker and colleagues ${ }^{12}$ found prolonged inconsolable or high pitched crying in $2.5 \%$ of infants who received DTP. We found this in 10 infants $(<1 \%)$. Hypotonic episodes were seen in two infants $(0.07 \%)$, which is less than that reported for DTP $(0.15 \%){ }^{13}$ The lower incidence of these events in our study may reflect batch variation of the DTP.

We also showed that a small number of infants $(<2 \%)$ immunised with the Men C vaccine along with other routine immunisations may develop fever, injection site reactions, rash, diarrhoea, gastroenteritis, anorexia, vomiting, somnolence, or rhinitis. Again, such adverse events are well known to be temporally associated with the administration of DTP and Hib vaccine without Men $\mathrm{C}^{13}$ and also occur in infants who have not been immunised. The only adverse events that could definitely be associated with the vaccine were reactions at the injection site, which occurred in four $(0.15 \%)$ of the 2796 infants.

Studies involving a large number of subjects are necessary to detect the presence of uncommon serious adverse events associated with a vaccine. The only other study published to date which was large enough to identify uncommon adverse events associated with a Men $C$ vaccine was a safety and efficacy study involving a heptavalent pneumococcal vaccine. ${ }^{14}$ In a double blind trial design, 37868 infants were randomly assigned to receive either the pneumococcal vaccine or another Men C CRM 197 conjugate (Wyeth) vaccine at 2, 4, 6, and 12-15 months along with routine immunisations. A total of 18941 infants received one or more doses of the control meningococcal vaccine. Safety analysis has not revealed any severe adverse events related to the vaccination and the local and systemic reactions observed were generally mild. ${ }^{14}$

This is the only study published to date designed exclusively to identify at least one uncommon adverse event associated with this Men $\mathrm{C}$ vaccine in infants. Other studies involving smaller sample sizes have looked at the reactogenicity of the Chiron Men $\mathrm{C}$ vaccine and other Men $\mathrm{C}$ vaccines in infants and found them to be well tolerated. ${ }^{2-7}$ The methodology of these studies was different from ours in that they involved recording of day to day health of the infants for about a week after the vaccination in a diary designed for this purpose. Subsequent to this study and partly based on its results, this vaccine was licensed in the UK for infants on 13 October 2000. It was licensed for use in children aged 1 and older on 6 March 2000.

In November 1999, the Department of Health in the UK introduced a programme to immunise all individuals from birth to 18 years of age with conjugate meningococcal $\mathrm{C}$ vaccines. Postlicensure surveillance was by the Medicines Control Agency (MCA) using the "yellow card" system. By 1 June 2000 the MCA had received 4764 reports of reactions to the Men $\mathrm{C}$ vaccine, a reporting rate of one per 2875 distributed doses. The Committee on Safety of Medicines (CSM) reviewed all available data in June 2000 and found that reported suspected reactions (including headache, dizziness, nausea, faints, fever, rash, injection site reactions, vomiting, diarrhoea, irritability, crying, malaise, drowsiness, myalgia, lymphadenopathy, allergic reactions, impaired sleeping, and anorexia) were very rare, with none of the reactions being reported at a frequency of greater than one in 10000 distributed doses, with similar profiles for the Chiron and Wyeth vaccines. ${ }^{15}$ The advantage of the much larger 
numbers contributing to this postlicensure surveillance has to be weighed against the passive and incomplete nature of the reporting. Their data collection spans all age groups and is not easily compared with the active prospectively monitored adverse event data from our study.

The need for adequate, robust safety data to protect children and to avoid the potentially disastrous effects on public confidence in immunisation programmes of introducing an unexpectedly dangerous vaccine has to be balanced with the need to introduce vaccines into the general population in a timely fashion to prevent deaths and morbidity and with the cost and logistics of conducting such studies. The Men $C$ vaccine programme was unusual in that large scale efficacy studies, the usual vehicle for obtaining safety data, were not undertaken. It is likely that the ethical and practical dilemmas reflected in the design and conduct of studies such as this will be encountered again in the future if childhood vaccines against other rare but serious infections are developed.

A total of 78 practices in Sheffield and neighbouring communities and 87 practices in Scotland participated in this study- too numerous to list here. However, we thank their partners and staff and the staff at the community pharmacies who worked on this study as well as the parents and families and the children themselves who took part.

1 Public Health Laboratory Services (PHLS) website. www. PHLS.co.uk

2 Rennels MB, Edwards KM, Kayserling HL, et al. Safety and immunogenicity of four doses of Neisseria meningitidis immunogenicity of four doses of Neisseria meningitidis infants. Pediatr Infect Dis ₹ 2001;20:153-9.
3 English M, MacLennan JM, Bowen-Morris JM, et al. A randomised, double-blind, controlled trial of the immunogenicity and tolerability of a meningococcal group C conjugate vaccine in young British infants. Vaccine 2000;19: 1232-8.

4 Richmond P, Borrow R, Miller E, et al. Meningococcal serogroup C conjugate vaccine is immunogenic in infancy and primes for memory. F Infect Dis 1999;179:1569-72.

5 Bramley JC, Hall T, Finn A, et al. Safety and immunogenicity of three lots of meningococcal serogroup $\mathrm{C}$ conjugate vaccine administered at two, three and four months of age. vaccine administered at two

6 MacLennan JM, Shackley F, Heath PT, et al. Safety, immunogenicity, and induction of immunologic memory by a erogroup C meningococcal conjugate vaccine in infants: a randomized controlled trial. $\mathscr{f} A M A$ 2000;283:2795-801.

7 Richmond P, Borrow R, Findlow J, et al. Evaluation of de-O-acetylated meningococcal $\mathrm{C}$ polysaccharide-tetanus toxoid conjugate vaccine in infancy: reactogenicity, immunogenicity, immunological priming and bactericidal activity against $\mathrm{O}$-acetylated and de-O-acetylated serogroup C ity against $\mathrm{O}$-acetylated and de-O-acetylat

8 Ramsay M, Andrews N, Kaczmarski EB, Miller E. Efficacy of meningococcal serogroup c conjugate vaccine in teenagers and toddlers in England. Lancet 2001;1(357):195-6.

9 Rennels MB. The rotavirus vaccine story: a clinical investigator's view. Pediatrics 2000;106:123-5.

10 MacDonald NE, Halperin SA, Law BJ, et al. Induction of immunologic memory by conjugated vs plain meningococcal $C$ polysaccharide vaccine in toddlers: a randomized controlled trial. $\mathcal{F A M A}$ 1998;280:1685-9.

11 Choo S, Zuckerman J, Goilav C, et al. Immunogenicity and reactogenicity of a group $\mathrm{C}$ meningococcal conjugate vaccine compared with a group $\mathrm{A}+\mathrm{C}$ meningococcal polysaccharide vaccine in adolescents in a randomised observerblind controlled trial. Vaccine 2000;18:2686-92.

12 Decker MD, Edwards KM, Steinhoff MC, et al. Comparison of 13 acellular pertussis vaccines: adverse reactions. Pediatrics 1995;96:557-66.

13 Tinnion ON. Acellular vaccines for preventing whooping cough in children. Cochrane Database of Systematic Reviews $2000 ;(4): 2000$.

14 Black S, Shinefield H, Fireman B, et al. Efficacy, safety and immunogenicity of heptavalent pneumococcal conjugate vaccine in children. Northern California Kaiser Permanente Vaccine Study Center Group. Pediatr Infect Dis $\mathcal{F}$ 2000;19:187-95.

15 Department of Health. Chief Medical Officer's Update number 27. London: Department of Health, August 2000:4.

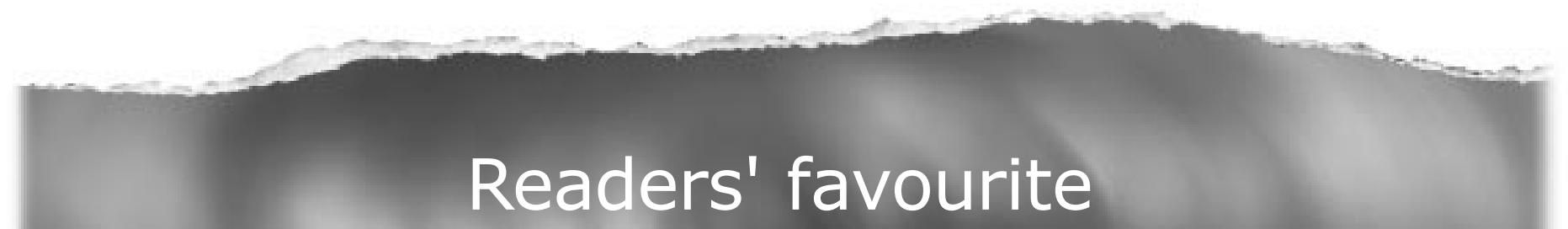

Top 10

Click on the "Top 10" button on the homepage

to see which are the best read articles each month

www.archdischild.com 